\title{
Flexural elasticity of woodpile lattice beams
}

\author{
Enrique Cuan-Urquizo ${ }^{\mathrm{a}, *}$, Atul Bhaskar ${ }^{\mathrm{a}}$ \\ ${ }^{a}$ Computational Engineering and Design Group, Faculty of Engineering and the \\ Environment, Boldrewood Innotavion Campus, University of Southampton, Burgess Road, \\ Southampton SO16 7QF, United Kingdom
}

\begin{abstract}
Flexure of slender structures, composed of filaments in a woodpile arrangement, is theoretically studied. Expressions for the apparent bending stiffness are derived. The model is validated experimentally using three-point bending. Computer simulations show that bending is accompanied by lattice shear for increased porosity. A shear-inclusive micromechanical model for two different stacking arrangements is developed. This is further refined by including shear deformation within the filaments, which is supported by numerical simulations for relatively short filament overhang. The apparent flexure and shear of lattice beams are attributed primarily to stretch and flexure of the filaments, respectively. Asymptotic formulas for the effective bending stiffness in terms of the relative density are presented. The apparent bending stiffness scales linearly with the volume fraction, whereas the apparent shear stiffness scales with the cube of the volume fraction. Exclusion of lattice shear leads to errors for by over an order of magnitude in the extreme, unlike void-free solid beams where shear is a higher order effect. Excellent quantitative agreement with numerical results is obtained for a wide range of porosity and external aspect ratio, when lattice shear is included in the model.
\end{abstract}

Keywords: structure-property relationship, flexural properties, apparent shear modulus, woodpile lattice

2010 MSC: 00-01, 99-00

\footnotetext{
* Corresponding author

Email address: ecuanurqui@itesm.mx (Enrique Cuan-Urquizo)
} 


\section{Introduction}

Materials with well defined internal architecture are increasingly becoming practically realisable; an interesting technology enabling this is that of additive manufacturing. This has stimulated fundamental research in the area of mechanics inspired by a host of technological applications, where regular arrangement of matter can be exploited to achieve certain intended mechanical performance, not possible with homogeneous matter. For example, the field of elastic meta-materials is of great current scientific interest. Here we are concerned with the apparent elastic behaviour of structures made of porous lattices in the so-called woodpile arrangement when they are subjected to flexure. This arrangement of matter is easy to fabricate using additive manufacturing and is found in a number of applications such as catalyst immobilization structures [1, 2, photonic crystals [3, 4, 5], lattice filters in ferrous casting [6] and tissue engineering scaffolds [7, 8, 9]. In the use of fused deposition modelling (FDM), the woodpile arrangement appears naturally during manufacturing. In this lattice architecture, layers of cylindrical filaments are stacked with alternating layers that contain filaments oriented in orthogonal directions, in a manner similar piles of wooden logs. By engineering the structure of the woodpile arrangement, the response can be tailored in a certain way. For example, the bandgap can be modified as required by changing the separation between filaments and their periodicity [4, 5]. In tissue engineering, the cell proliferation is affected by the interconnected channels formed from the distribution and stack of filaments [10]. Structural parameters such as, the separation of filaments, have shown to have a strong influence on the mechanical properties of FDM components [11, 12. These properties haven been studied primarily under tensile [13] and compressive loadings [14. However, transverse bending of microstructured slender structures is of great practical interest because of the loading scenario is frequently encountered, such as those within sandwich constructions.

Experimental approaches have been taken in the past to study the mechani- 
cal properties of FDM-components [11, 15]. Ahn et al. [11] reported anisotropy resulting from the preferred orientation of filaments in FDM materials in an experimental study. Bellini and Guceri [15] experimentally characterised materials fabricated using fused deposition modelling for various raster sequences with a view to determine the anisotropic elastic constants from measurements. Montero et al. [12] used the design of experiments approach to study the influence of deposition strategies on the strength of FDM-samples. Too et al. [16 fabricated similar structures using FDM and observed rapid reduction in strength when porosity is increased. Tymrak et al. [13] characterised the mechanical properties from the tensile testing of samples fabricated using FDM. Both Sood et al. 17] and Onwubolu and Rayegani [18 built empirical models to predict the strength of FDM-samples based on design of experiments. Wu et al. [19] fabricated PEEK-based materials using 3D-printing and experimentally characterised their strength for various raster angles of the FDM filament orientation. Croccolo et al. 20] experimentally studied the role of filament orientation $\left(45^{\circ}\right)$ upon tensile stiffness and strength for fused deposition fabricated parts and also provided an analytical model based on the mechanics of inclined filaments.

The woodpile architecture provides interconnected voids effectively when used in tissue engineering (Yeo et al. 21]). The mechanical properties of such arrangment have been studied in this context. Norato and Wagoner-Johnson 22] studied a woodpile lattice of hydroxyapatite filaments fabricated using robocasting. They proposed an analytical model for the elastic response along the stacking direction. The model was derived by approximating the elastic response of the filaments under compression with that of a disc under diametrical compression. Naghieh et al. [23, studied elastic response by means of Finite Element Analysis and compression tests on polylactic acid (PLA) samples fabricated using fused deposition modelling. Serra et al. 24 fabricated PLA-based porous biomaterial using 3D printing for a few alternatives of woodpile arrangements emphasizing the role of microstructure on mechanical properties of such scaffold materials and cell response therein. Ceramic scaffolds with lattice architecture have been studied for load-bearing applications in the context of bone 
tissue engineering (Cesarano et al. [25]). Martinez-Vazquez et al. 26] fabricated ceramic scaffolds having woodpile internal architecture to enhance cell attachment, proliferation and growth and presented a finite element simulation relating applied nominal stress and the maximum stress within the filaments.

Despite huge practical interest, attempts to understand the flexural response of beams made of such lattice material are rare, with the exception of a few recent works [27, 28, 29, 30, 31. Martinez-Velazquez et al. [27. studied the flexural properties of $\beta$-tricalcium phosphate based samples experimentally in four-point bending. The influence of the impregnation of the samples with polymers such as polylactic acid and poly( $\varepsilon$-caprolactone) was studied in 27. Liu et al. 28 characterised bioactive glass scaffolds by mechanical testing for compression, flexure and fatigue. The effect of incorporating polylactic acid and poly( $\varepsilon$-caprolactone) on the mechanical properties of bioactive glass scaffolds was studied by Eqtesadi et al. 29. Malinauskas et al. 31] measured the flexural modulus from three-point bending tests on FDM samples with different woodpile arrangements. Domingo-Espin [30] studied L-shaped parts under combined bending and torsional loading both computationally and experimentally. However, no attempt to predict the flexural response of slender woodpile structures exists in the published literature.

A fundamental research question is to understand the structure-property relationship of material with structured porosity. Gibson and Ashby related structure to apparent Young's modulus for two dimensional hexagonal honeycombs 32. However, due to considerable differences in the architecture of honeycombs vs that of the woodpile, such results for $2 \mathrm{D}$ lattices cannot be used for woodpile lattices. A lattice consisting of composite titanium wires was studied by Moongkhamklang et al. [33, 34]. In [33, the apparent compressive properties, when the sample is loaded along the filaments, were studied experimentally. However, the geometry considered by them- one of a square lattice deformed in its plane- is significantly different from that of the woodpile. The apparent shear response was identified to be for in-plane shear of square lattice 34. The present work, by contrast, is concerned with the stacks of filaments that bend 
and shear while being separated by similar stacks running orthogonally but not in the same plane and bent out of these planes. The geometry of the problem combined with the loading do not appear to have been considered theoretically before. Slender structures under transverse loading are commonly used as structural components in a variety of non-biomedical contexts. The effective elastic properties of 2D structured beams was studied by Banerjee and Bhaskar [35, 36, 37. They characterised the effective properties of porous media having hexagonal, triangular and irregular cells via the dynamics of cellular beams.

The review of the existing literature suggests that theoretical models for the transverse response of slender structures made of lattice materials that make use of filaments as the fundamental manufacturing element stacked in the woodpile configuration, are missing. This paper is motivated by this gap in the fundamental understanding and is arranged as follows: In Section 2 analytical models for the apparent properties of the lattice material having woodpile architecture are developed. Expressions for the apparent Young's modulus, bending stiffness and shear stiffness are derived. The model is experimentally validated using three-point bending of lattice structures, starting from single filament tensile tests. Further numerical results and discussions are presented in Section 3 where transverse deflection of lattice beams accounting for various mechanisms of fibre deformation are considered. Concluding remarks are given in Section 4

\section{Transverse deflection of woodpile lattices and flexure of slender beams}

Analytical models for the apparent elastic properties of the lattice material in woodpile arrangement, such as those manufactured using fused deposition modelling, are developed in this section. The cross-section of FDM fabricated material, a schematic diagram of such woodpile lattice, and a unit cell of such a periodic arrangement are shown in figures 1a, 1p, and 1 respectively. Parallel filaments are stacked so they run orthogonally within alternate layers. Cantilever beam arrangement provides a typical bending scenario as shown in figure 


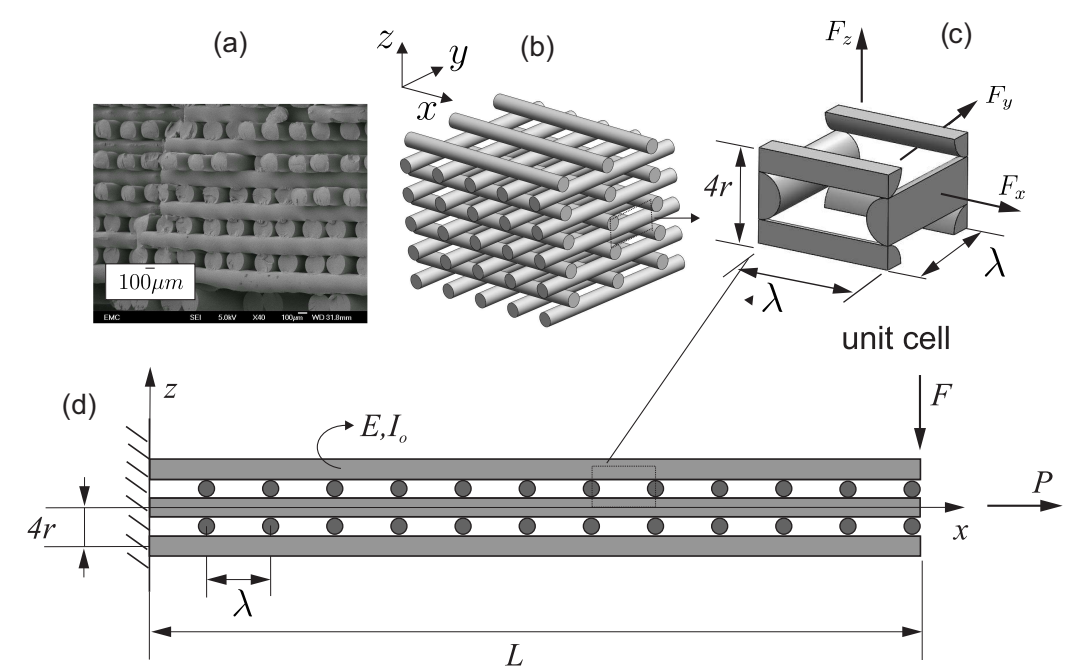

Figure 1: (a) SEM micrograph of a FDM fabricated woodpile structured material (b) A woodpile lattice with filaments along $x$ - and $y$-axes. The stacking direction is $z$. (c) A repetitive unit within an infinite lattice, (d) a woodpile beam under combined axial and transverse loading.

11. Only five layers of filaments stacked over each other are shown for the ease of illustration. A combined axial force $P$ and a transverse bending force $F$ at the tip are shown. We will consider each of these two in turn.

The analysis presented here has some limitations that need to be spelt out. Firstly, the cubic symmetry of the lattice geometry would result in anisotropy of the apparent elastic properties. In particular, the material would be orthotropic because of the symmetry about three orthogonal planes, in addition to the fact that properties are vastly different in the stacking direction $z$ than the other two directions along the $x$ - and the $y$-axes, i.e. parallel to the filaments because the stacking direction elasticity is dominated by either flexure or compression of the filament one over another, depending on whether the stacking within alternate layers is "staggered" or "eclipsed". These consideration are beyond the scope of the present paper because the analysis is involved 38. Note that even if properties in the two filament direction are the same, there exists an in-plane anisotropy, i.e. properties would change upon changing the orientation within the plane. Here we restrict ourselves to flexure when filaments are oriented 
along and across the bending axis as shown in figure 1 .

Anisotropic off-axis flexure could still be studied using a unit cell analysis, but that would require determination of all elastic constants before coordinate transformation of the rank-4 elasticity tensor could be effected. The present work does not address these and will be a subject of future studies. Models presented in Sections 2.4 and 2.5 are restricted to the linear elastic limit. Compressive behaviour along the fibre direction could involve filament buckling-another exclusion from the present work. Finally, uniformity of the lattice spacing is assumed, any imperfection ignored, and any misalignment of the filaments to the loading direction-present in real scenarios-is ignored.

The circular cross-section of the filaments has area $a$, radius $r$, and the second moment of area $I_{o}$ about its centroid. The separation between filaments within a layer is $\lambda$ in both $x$ - and $y$-directions whereas that across the layers between axes of fibres belonging to alternate layers having the same filament orientation is $4 r$ (see figure 1 1 ). Young's modulus of the parent material is denoted by $E$, the shear modulus is $G$ and Poisson's ratio is $\nu$.

2.1. The apparent Young's modulus along the filaments and its relevance to flexure

Consider a piece of woodpile lattice material as shown in figure 2, when viewed along the stacking direction ( $z$-direction in figure 1p). A remote stress $\sigma_{\infty}$ is applied along one of the two principal fibre directions. The results for the apparent stiffness are well-known [34. We include them here (i) for completeness, and (ii) to examine their usefulness for more complex loading such as that encountered in beam bending, The apparent Young's modulus along the filament direction $\langle E\rangle$, after elementary calculations which assumes that filaments running across the loading direction do not participate, is obtained as

$$
\langle E\rangle=E \phi
$$


where the angular brackets \langle\rangle represent the relevant apparent property and $\phi$ is the fractional cross-sectional area exposed to tensile loading. Expression (1) can also be expressed in terms of relative density of porous material 34

$$
\langle E\rangle=(1 / 2) E \bar{\rho}
$$

where the relative density $\bar{\rho}$ is equal to the volume fraction $\bar{\rho}=V_{s} / V_{c}$. Here $V_{s}$ is the volume occupied by the filaments, and $V_{c}$ is the total apparent volume of the porous material. The volume of the solid within the repetitive unit occupied by the fibres is given by $V_{s}=2 \pi r^{2} \lambda$. The total volume of the rectangular cuboid is $V_{c}=4 r \lambda^{2}$. The relative density is then given by $\bar{\rho}=V_{s} / V_{c}=\pi r / 2 \lambda$, so that $\langle E\rangle=(\pi r / 4 \lambda) E$.

Note the factor of $(1 / 2)$ in the expression for $\langle E\rangle$ in equation (2) which, in the limit of $\bar{\rho} \rightarrow 1$, becomes $\langle E\rangle \approx(1 / 2) E$. This seems to be inconsistent with the expectation that $\langle E\rangle \rightarrow E$ in the limit of dense solid. The apparent discrepancy is resolved when we realise that half of the material within fibres running orthogonally to the loading direction do not participate. When the volume is imagined to be filled completely, in the limit $\bar{\rho} \rightarrow 1$, this would not be the case. Thus the validity of expression (2) is limited to $\bar{\rho}<1$ and when parallel filaments are well separated. A linear dependence of $\langle E\rangle$ on $\bar{\rho}$, as exhibited by equation $[2]$, i.e. $\langle E\rangle \sim \bar{\rho}$, is expected because micromechanics involving stretch of filaments leads to stiffness that scales linearly with characteristic cross-sectional area, which, in turn, is proportional to the volume fraction.

A frequently encountered loading scenario for slender structures is that bending. Since bending results in the stretch of fibres running parallel to the axis of lattice beams, it is reasonable to make use of the apparent elastic modulus $\langle E\rangle$, given by equation (2), and be able to predict the flexural response. The rest of the paper is concerned with the effectiveness and the limitations of this approach and the inclusion of further important effects unaccounted for. 




(a)

(b)

Figure 2: Schematic diagrams of (a) the lattice material subjected to remote uniaxial stress $\sigma_{\infty}$, and (b) a single strut showing deformation mechanism under this load.

\subsection{The use of the apparent Young's modulus in flexural response calculations}

The usefulness of the apparent Young's modulus in calculating the bending response of lattice beams with filaments running parallel to the axis of the beam is immediate, if we assume the scale of porosity much smaller than the characteristic length in the cross-section of the beam, and also if we ignore shear within the lattice. Under these simplifications, the apparent bending stiffness of the lattice beam is given by

$$
\langle B\rangle=E\langle I\rangle
$$

where $\langle I\rangle$ is the second moment of the area of the filaments about the centroidal axis. Therefore,

$$
E\langle I\rangle=E I_{\text {filaments }}=E \int z^{2} \mathrm{~d} A_{\text {filaments }}
$$

But $\mathrm{d} A_{\text {filaments }}=\phi \times \mathrm{d} A$, where $\mathrm{d} A$ is an area element over the cross-section of the lattice beam smeared as equivalent homogeneous beam, and where $\phi$ is the fraction of the cross-section occupied by the filaments. Since all the filaments have the same properties, $E$ is independent of the filament in question, so we have

$$
E\langle I\rangle=\int(E \phi) z^{2} \mathrm{~d} A
$$


where the integral is to be carried out over the complete smeared area of the cross-section having voids filled with equivalent modulus material. Further, assuming that porosity is independent of the distance away from the neutral axis, i.e. $\phi \neq \phi(z)$ and because $E \phi=\langle E\rangle$, we have

$$
E\langle I\rangle=\langle E\rangle \int z^{2} \mathrm{~d} A=\langle E\rangle I
$$

where the integral in the above equation is over the apparent porosity-filled area of the rectangular cross-section of the scaffold structure and $I$ is the second moment of area of the same about the centroid of the smeared area. Therefore, the bending stiffness equals the Young's modulus of the fibre material $E$ times the apparent second moment of the area of the filaments over the cross-section, which also equals apparent tensile modulus $\langle E\rangle$ times the second moment of porosity filled cross-sectional area. This analysis employs simplifications that the lattice dimensions are small so that porosity (quantified by the area fraction $\phi)$ could be treated as a continuous variable independent of $z$; and also other deformation mechanisms of the filaments are ignored. These effects could be significant and also the role of finiteness of the number of stacks could be important, so we may not be able to treat porosity as a uniform variable over the cross-section. The role of finiteness of the number of stack is taken up in the next sub-section where expressions for the scaling of the bending stiffness with porosity are also developed in the asymptotic limit of large number of stacks.

\subsection{Flexure of woodpile structures: apparent bending stiffness}

Consider the flexure of a slender woodpile beam subjected to remote bending moment $M_{\infty}$ as shown in figure 3 . Each filament induces a moment about the neutral axis (NA) of the lattice beam as it elongates or shortens according to its distance from the NA. Therefore, the apparent bending stiffness $\langle B\rangle$ of the lattice structure can be expressed as $E\langle I\rangle$, where $\langle I\rangle$ is the effective second moment of area of the filaments distributed over the beam cross-section as given by the parallel axis theorem. The stress distribution within the filaments is shown 
in figure $3 \mathrm{~b}$ which is a linear variation in a accordance with Euler-Bernoulli hypothesis for thin beams. Note that the bending moment induced at the neutral axis of the lattice beam is dominated by the stretch of the filaments rather than their flexure. The moment at the neutral axis due to stretch of the filaments is proportional to the distance from NA. As we go away from the neutral axis of the lattice structure, the relative importance of filament bending is diminished with respect to filament stretch. Even when the remote stress is tension-free, i.e. pure bending, the loading on each filament at a distance $D$ from the neutral axis is a combination of tension $T$ and bending moment $M$, as shown in figure 3.

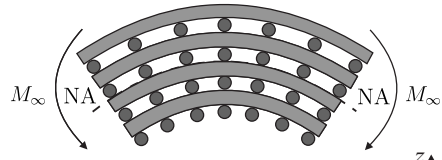

(a)



(b)

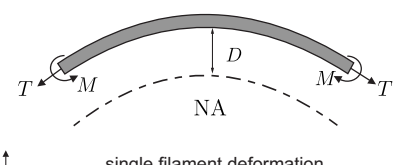

Figure 3: Schematic diagrams of (a) the lattice material subjected to remote bending moment $M_{\infty}$, and (b) stress distribution in the woodpile beam in flexure, showing the filaments above the NA in tension and the ones below the NA in compression. (c) A single filament under tension $T$ and bending moment $M$.

When beams made of lattice material are bent in a plane as shown in figure 3a, the displacement field within every stack of filaments, separated in the direction perpendicular to the plane of the paper, would appear to be identical. Also the filaments running perpendicular to the plane of the paper in figure $3 \mathrm{a}$, where they appear circular in their end views, would participate in the elastic deformation minimally. This allows us to simplify consideration of the lattice geometry significantly. Modelling just one such group of curved filaments together with those providing spacing between them would be adequate. Such a simplification is shown in figure 4 for odd number of stacks of filaments that participate in bending (filaments of darker shade).

Treating each filament as a beam, the apparent second moment of area, for an odd number of layers, is obtained by summing contributions from each, while accounting for the distance away from the neutral axis of the stack of filaments. 


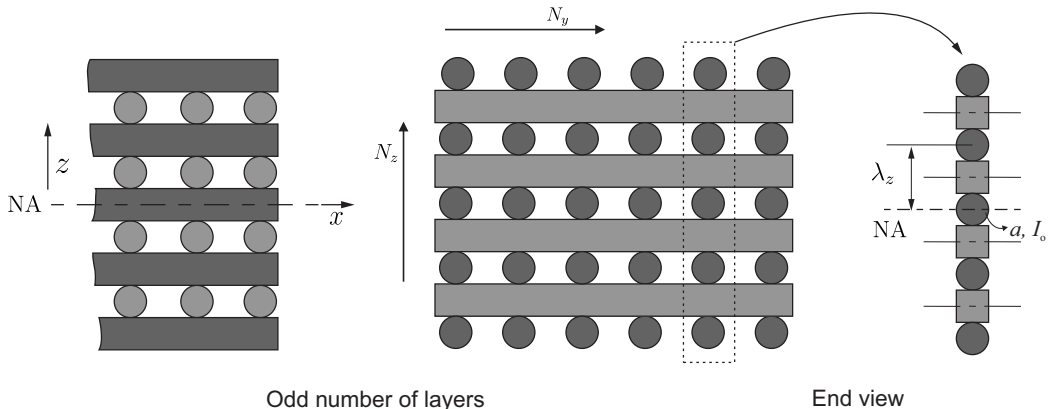

Figure 4: Schematic diagram of the cross-sections of a lattice beams with odd number of layers.

Using the parallel axis theorem, we obtain the apparent second moment of the cross-sectional for a stack of filaments with circular cross-section as

$$
\langle I\rangle=2 N_{y}\left[\sum_{i=1}^{\left(N_{z}-1\right) / 2}\left(I_{o}+16 i^{2} a r^{2}\right)+\frac{I_{o}}{2}\right] .
$$

Here $N_{z}$ is the number of layers in the stacking direction (figure 4), $N_{y}$ is the number of such groups of filament in the $y$-direction, $4 r$ is the separation between layers, $I_{o}$ is the second moment of area of each filament about its own centroid. The upper limit of summation $\left(N_{z}-1\right) / 2$ accounts for contribution on one side of the neutral axis; the factor 2 ensures the sum is carried out above and below the neutral axis. The last term within the brackets is the contribution of the filament at the centre of the stack. An expression for even number of vertically stacked layers is similarly obtained as

$$
\langle I\rangle=2 N_{y}\left[\sum_{i=0}^{\left(N_{z}-2\right) / 2}\left(I_{o}+a\left(\frac{4 r}{2}+4 i r\right)^{2}\right)\right] .
$$

Expressions in both (7) and (8) have the leading order terms that scale as $\sim \sum i^{2}$. The next most significant term in $(8)$ has a term that scales as $\sim \sum i$. The first of these can be summed up analytically using the square pyramidal number formula. The term $\sim \sum i$ in $(8)$ is an arithmetic series that can also be summed up analytically. Further, using the filament cross-sectional area $a \sim r^{2}$ 
in the expansion of these and reorganising terms, we obtain a single expression valid for odd, as well as even, number of stacks of filaments as

$$
\langle I\rangle=8 \pi r^{4} N_{y}\left(\frac{1}{6} N_{z}^{3}-\frac{13}{96} N_{z}\right) .
$$

Therefore $\langle I\rangle \sim r^{4}$ which is consistent with: (i) the filament bending stiffness about its own centroid of the circular section scales as $I_{o} \sim r^{4}$, and (ii) the stretch of the filaments induce a second moment of area about the neutral axis of the lattice beam proportional to area $a$ times the square of separation. Since area $a \sim r^{2}$ and the separation $\sim r,\langle I\rangle \sim r^{4}$. Further $\langle I\rangle \sim N_{z}^{3}$ holds when $N_{z} \gg 1$ so that the first term within the parenthesis dominates and we have an asymptotic formula for large $N_{z}$ as $\langle I\rangle \approx(4 / 3) \pi r^{4} N_{y} N_{z}^{3}$.

The apparent bending stiffness can now expressed in terms of the volume fraction of the porous lattice $\bar{\rho}$, in the asymptotic limit of small lattice separationrelative to the external dimensions of the scaffold cross-section-as

$$
\langle B\rangle=C \bar{B} \bar{\rho}^{n}
$$

where $\bar{B}$ is a reference bending stiffness taken as

$$
\bar{B}=\frac{E}{12}\left(N_{y}-1\right)\left(N_{z}-1\right)^{3} \lambda(4 r)^{3}
$$

which is the second moment of the porosity-filled cross-sectional area, making $\bar{B}$ as the bending stiffness of a dense beam. Using equation (9), and recognising that $\langle B\rangle=E\langle I\rangle$, we obtain

$$
C=\frac{N_{y}\left(N_{z}^{3} / 2-13 N_{z} / 32\right)}{\left(N_{y}-1\right)\left(N_{z}-1\right)^{3}} \quad \text { and } n=1
$$

i.e. $\langle B\rangle \sim \bar{\rho}$. The linear scaling of bending stiffness with the volume fraction is in agreement with $\langle E\rangle \sim \bar{\rho}$ obtained in Section 2.1. This is consistent with the axially running filaments being predominantly in tension and compression. 


\section{Experimental validation by three-point bending tests}

The model for bending stiffness developed in the previous sub-section is now validated experimentally. Lattice structures composed of woodpile arrangement were additively manufactured for testing under three-point bending. Bespoke computer scripts were developed to generate G-codes that drive the 3D printer tool path in a controlled way in order to create samples of desired lattice geometry while following ASTM D790 standard for the external sample shape. FDM $3 \mathrm{D}$ printer $B F B$ touch 3000 printer $\AA$ was used to manufacture the experimental samples using white PLA filament from the manufacturer KDI Polymer Specialists Ltd $\AA$. The nozzle diameter is $2 r=0.5 \mathrm{~mm}$ with a traverse displacement speed of $860 \mathrm{~mm} / \mathrm{min}$. The filaments within the printed samples run parallel to the $x$ - and $y$-axes of the machine, whereas thickness of the samples is along the $z$-axis. The spacing between filaments was kept as $\lambda=1 \mathrm{~mm}$ and the distance between layers as $\lambda_{z} \approx 4 r=1 \mathrm{~mm}$. INSTRON 5569 with a $2 \mathrm{kN}$ load cell was used for testing. Rollers of $8 \mathrm{~mm}$ diameter were selected for applying the transverse force and also as supports placed at a distance of $88 \mathrm{~mm}$ according to the ASTM D790. The machine was set in the displacement control mode with a maximum displacement of $20 \mathrm{~mm}$ at a strain rate of $5 \mathrm{~mm} / \mathrm{min}$. The force and displacement data were recorded and exported for further processing.

An image of a typical sample under testing is shown in figure 5 a; the red arrows indicate the rollers and loading direction. A schematic diagram of the same arrangement appears in figure 5b. Load-deflection measurements for ten identically produced samples are presented in figure 55. The response in bending is composed of a clear linear elastic part, followed by a non-linear region. The nonlinearity is attributed to plasticity as well as kinematic nonlinearity-the latter being recoverable upon unloading. The initial part of the curve starts to flatten at deflection $\approx 3 \mathrm{~mm}$. The samples resisted a maximum load of $\approx 130$ $\mathrm{N}$ at a transverse displacement of $8 \mathrm{~mm}$. Beyond this, the filaments of the bottom layer are under tensile stress, experienced necking followed by failure due to fracture. Here we are interested only in the apparent stiffness of such 

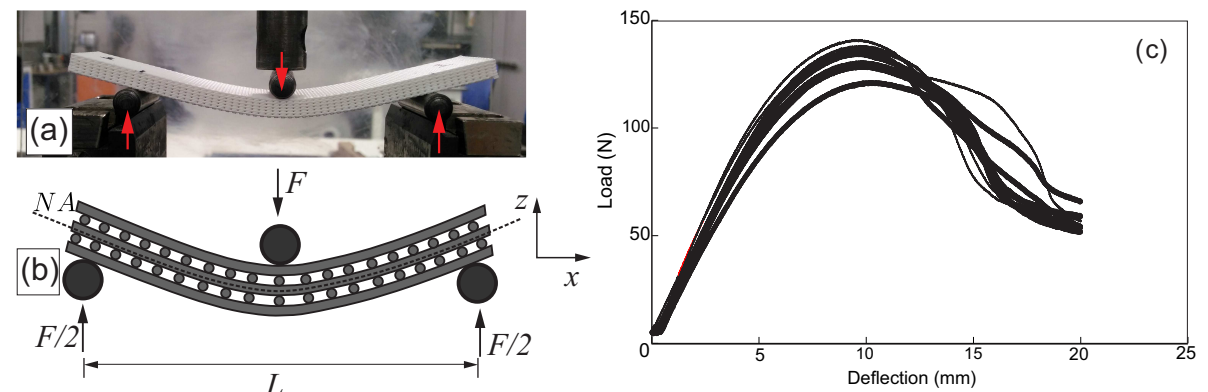

(d)


Figure 5: (a) Photograph of a sample during three-point bending test, (b) the schematic showing the filaments deformation mechanisms and loading direction, and (c) the force-deflection curves measured for the whole set of samples. (d) DEBEN $(\mathbb{R}$ micro-tester device showing the load direction and (e) a single filament after failure.

structures, hence only in the slope of the linear part of the measured response.

Expressions relating the bending stiffness of the scaffold to the lattice structure require properties of the filament material. Since the filaments are melt extruded during the additive manufacturing process, reliable estimate of their material properties should be assessed from direct measurement in order to validate theoretical models. Therefore, filaments were melt extruded under identical conditions of printing (e.g. temperature, fan, extrusion speed) as those for used for the manufacture of the lattice. They were then tested in tension using DEBEN $\AA$ micro-tensile test stage that consists of two clamps and is actuated with a motor and threaded load screw that moves one of the clamps linearly and the force measured with a 300N load cell (see figure 5 $\mathrm{d}$ ). A typical test sample is shown in figure 5 5 . The Young's modulus of the filament material was estimated as $E=2290 \pm 315 \mathrm{MPa}$, averaged over 11 such filament samples.

We can now use equation (9) to calculate the deflection $\delta$ for three-point bending, $\delta=F L^{3} / 48\langle B\rangle$; incorporating experimentally measured filament ma- 




Figure 6: Linear elastic part of the load-displacement curves from the 3-Point-Bending tests, the mean of these data and the predicted trend. The error bars are shown at 1 standard deviation from the experimental mean.

terial property $E=2290 \mathrm{MPa}$ (the mean value) and the overhang between the supports $L=88 \mathrm{~mm}$. The samples tested had 12 layers, 6 layers of filaments running axially and 6 running across $N_{z}=6$. Each layer has $N_{y}=26$ axially running filaments. The number of filaments running across the axis of the beam is 124. The cross-sectional area of each filament was measured using a Makroscope M420 $a=0.302 \mathrm{~mm}^{2}$ which is averaged over 11 samples. The effective second moment of area was calculated using equation $(9)$ as $\langle I\rangle=137.889$ $\mathrm{mm}^{4}$. The load-deflection measured curves and the theoretically calculations show excellent agreement (figure 6). A systematic trend that the theoretical values of stiffness are slightly higher than the measured stiffness is observed. This is attributed to neglecting shear deformation in the model. The effect of shear is progressively more pronounced as the lattice spacing increases. This warrants refinement of the model which is taken up next and reconciled with numerical experiments in Section 3.

\subsection{The role of shear in transverse deflection of woodpile beams}

When a slender solid beam is subjected to transverse loading, the transverse deflection scales according to $\delta \sim\left(L^{3} / E_{s} I\right)$, where $L$ is the overall length of the beam and $E_{s} I$ its bending stiffness. The subscript $s$ indicated that the 
property corresponds to the solid beam. For relatively shorter solid beams, as well as for large lattice spacing, shear deformation needs to be accounted for [39, 40. In addition to slenderness and the shape of the cross-section, the relative importance of bending and shear in transverse deflection of solid beams depends on the shear modulus of the material $G_{s}$. For solid material, $E_{s} / G_{s}=2\left(1+\nu_{s}\right)$ is a fairly insensitive function of the Poisson's ratio $\nu_{s}$ of the material because of the narrow range of its values for commonly found materials. Therefore, for dense beams, the relative importance of bending over shear is primarily determined by the slenderness ratio and is relatively insensitive of the material properties. It is a higher order effect for slender void-free beams. By contrast, for lattice beams, the ratio of bending and shear properties could be extremely sensitive to the architecture of the lattice and the level of porosity because the apparent properties of lattices depend not only on the parent material properties but also the lattice architecture.

Consider a lattice under remote shear stress $\tau_{\infty}$, as sketched in figure $7 \mathrm{a}$. Bonding between struts is assumed to be at a point and it is assumed that the struts parallel to the $y$-axis do not contribute to the shear resistance and they do not rotate. The apparent shear stiffness is a consequence of the bending of the struts parallel to the $x$-axis alone. Shear within the cylindrical filaments, that appear circular on the $x z$-plane, could be ignored in the first instance; i.e. the circular shape is assumed not to be distorted during shear of the lattice. As opposed to this, shear in the filaments running parallel to the $x$-axis could be significant when the separation between the supporting filaments is short. Bonding between orthogonally oriented layers is assumed to be perfect. Therefore the shear of the woodpile is governed by filament transverse displacement $w$ in the $z$-direction as shown in figure $7 \mathrm{~b}$ for a repetitive unit. The dotted contour in figure $7 \mathrm{~b}$ represents the undeformed shape of the unit cell. A single filament, represented by a line, deformed between two consecutive points of bonding is shown in figure 7 .

In many additively manufactured scaffolds [41, 42, the diameter to overhang ratio $2 r: \lambda$ is frequently about $1: 2$. Therefore, thin beam theory may not be 


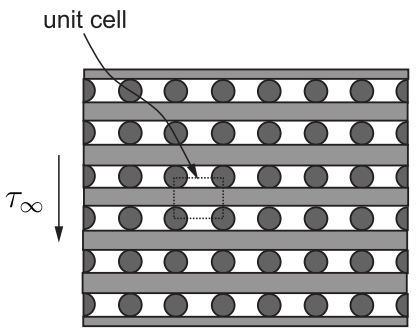

(a)



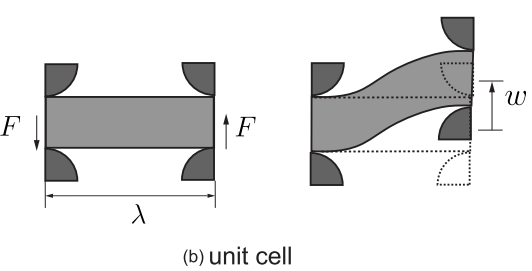

(b) unit cell

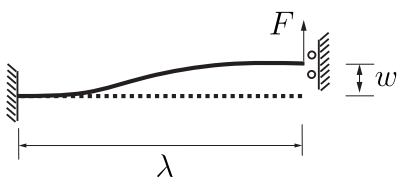

Figure 7: Schematic diagrams showing (a) the lattice subjected to shear stress $\tau_{x y}$, (b) a unit cell deformation due to the shear stress, and (c) the representative strut used for the analysis.

adequate to represent flexure of the filaments in many practical applications. Here we include shear deformation within the micromechanics of the filaments in addition to bending, in order to develop a reasonably accurate, yet simple model of woodpile lattice shear. Such a model would cover a much wider range of relative densities, including situations where shear deformation is significant. Applying shear correction in the spirit of Timoshenko, an expression for the tip displacement $w$ of a filament, such as the one shown in figure $7 \mathrm{p}$, can be readily derived. The boundary conditions applied to the bending equation are: (i) at $x=0$, deflection $=0, \varphi=0$ and (ii) at $x=\lambda$, deflection $=w, \varphi=0$, where $\varphi$ is the shear angle. The tip displacement as function of the applied force $F$ is thus obtained as

$$
w=F\left(\frac{\lambda^{3}}{12 B}+\frac{\lambda}{2 S}\right)
$$

where $B=E I_{o}$ is the bending stiffness of the filament, and $S=\kappa G a$ is the shear stiffness of the filament. Here, $\kappa$ is the shear coefficient for circular cross-sections describing shear within a filament. The tip displacement $w$ and the shear force $F$ can be related to the apparent shear stress $\tau_{x z}$ and apparent shear strain $\gamma_{x z}$ via

$$
\tau_{x z}=\frac{F}{A} \quad \text { and } \quad \gamma_{x z}=\frac{w}{\lambda}
$$

where $A=4 r \lambda$ is the total area of the unit cell over which the shear force acts. Combining (13) and 14 and defining the apparent shear modulus of the lattice 
as the ratio of the apparent remote shear stress and the apparent shear strain, i.e. $\langle G\rangle_{T}=\tau_{x z} / \gamma_{x z}$, we obtain

$$
\langle G\rangle_{T}=\frac{3 B S}{6 B r \lambda+S r \lambda^{3}} .
$$

The subscript $T$ indicates the inclusion of Timoshenko shear correction in filament bending. Equation (15) is implicitly an expression for the apparent shear modulus as a function of the parent material properties ( $E$ and $G$ ), and structural and geometrical parameters $(\lambda, r)$. The dependence of the flexural displacement $w \sim\left(\lambda^{3}\right)$ makes the apparent shear modulus very sensitive to the parameter $\lambda$. Using $B=E\left(\pi r^{4} / 4\right)$ and $S=\kappa G \pi r^{2}$, the expression for the apparent shear modulus is now obtained as,

$$
\langle G\rangle_{T}=\frac{3 \pi \kappa G E r^{3}}{6 E \lambda r^{2}+4 \kappa G \lambda^{3}} .
$$

The apparent shear modulus can be further expressed in terms of the relative density of the porous material. Using the relationship $\bar{\rho}=\pi r / 2 \lambda$, we have

$$
\langle G\rangle_{T}=\frac{\left(6 \kappa G E \bar{\rho}^{3}\right)}{\left(6 E \bar{\rho}^{2}+\kappa G \pi^{2}\right)}
$$

When Euler-Bernoulli model is used to describe the flexural mechanics of filaments, we obtain an expression for the apparent shear modulus of the woodpile lattice as

$$
\langle G\rangle_{E B}=\frac{6 E}{\pi^{2}} \bar{\rho}^{3}
$$

after carrying out algebra on the lines of equation through to (17) while ignoring the second term in 13 . The subscript $E B$ denotes Euler-Bernoulli bending model for the filaments as the basis of the derivation of the apparent shear property. The connection between the two expressions in equations (17) and 18 becomes clear when we divide the numerator and the denominator in 17 by $\kappa G$. In the limit of $\bar{\rho} \gg 1$, the two expressions coincide. This is expected as shear deformation theory represents the micromechanics better 
for relatively dense lattices, whereas it would provide negligible improvement over the apparent properties derived using Euler-Bernoulli theory for the micromechanics of thin filaments-a situation that corresponds to large porosity. Rearranging terms, we obtain $\langle G\rangle_{T}=\langle G\rangle_{E B} /(1+\epsilon)$, where $\epsilon=6 E \bar{\rho}^{2} /\left(\kappa G \pi^{2}\right)$. Using $E / G=2(1+\nu)$, we have $\epsilon=12(1+\nu) \bar{\rho}^{2} / \kappa \pi^{2}$, where $\nu$ is the Poisson's ratio of the filament material. For example, for the value of Poison's ratio $\nu=0.36$ [43, and shear coefficient of $\kappa=0.9$ for circular cross-sections [4], we obtain $\epsilon \approx 1.84 \bar{\rho}^{2}$. Using Taylor series expansion the apparent shear modulus including shear correction can be expressed as,

$$
\langle G\rangle_{T} \approx\langle G\rangle_{E B}\left(1-1.84 \bar{\rho}^{2}\right)+\mathrm{O}\left(\bar{\rho}^{4}\right)
$$

As an example, for $r / \lambda=1 / 5, \bar{\rho}=\pi / 10$, which gives $\langle G\rangle_{T} \approx 0.82\langle G\rangle_{E B}$, whereas for $r / \lambda=1 / 10,\langle G\rangle_{T} \approx 0.95\langle G\rangle_{E B}$ highlighting the importance of inclusion of shear within fibres for dense lattices - the correction required is over $18 \%$ for relative density $\bar{\rho}<0.3$. Also note the presence of the higher order term $\mathrm{O}\left(\bar{\rho}^{4}\right)$ whereas a term $\mathrm{O}\left(\bar{\rho}^{3}\right)$ is missing, which indicates a good approximation when we retain only the leading term $\mathrm{O}\left(\bar{\rho}^{2}\right)$ in the expansion the next significant term being much smaller. Expressions $(17)$ and $(18)$ for the apparent shear modulus of woodpile lattice materials are being reported for the first time.

\subsection{Shear of lattices with staggered filaments}

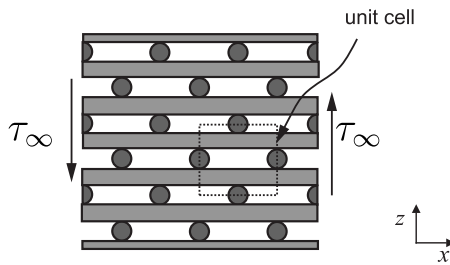

(a)

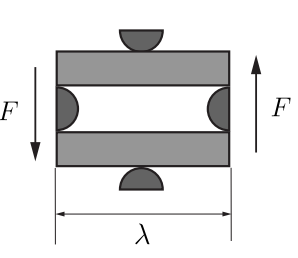

(b) unit cell



Figure 8: Schematic diagrams showing (a) the lattice subjected to shear stress $\tau_{x z}$, and (b) a unit cell deformation due to the shear stress.

Consider next the shear of a woodpile lattice with alternating layers in a stag- 
gered or 'eclipsed' arrangement as shown in figure 8 a. We are now interested in the transverse elastic response of beams made of such structured material. For bending in the $x z$-plane, the stiffness is a manifestation of tension and compression in filaments running parallel to the $x$-axis. Therefore, the expression for the apparent bending stiffness remains the same as that derived in Section 2.3 when the alternating layers in the stacking direction possess an either 'eclipsed' or 'aligned' arrangement - the relevant expressions being given by equations (9) through 12). We can now carry out analysis for shear of a lattice in the staggered arrangement of struts on the lines of that from equations 13 to 19 . Comparing the deflection profile of the filaments in figures (7) and (8), we note that the deflected shape of $(\lambda / 2)$ length in figure 8 is geometrically similar to the filament of length $\lambda$ in figure 7. The total vertical displacement $w$ of the unit cell is given $\frac{w}{2}=\frac{(F / 2)(\lambda / 2)^{3}}{12 B}+\frac{(F / 2)(\lambda / 2)}{2 S}$ which is similar to expression 13. except that half of the force over half of the overhang causes half of the total vertical displacement. After some algebra, the apparent shear modulus for the lattice in the staggered arrangement is obtained as function of the relative density as,

$$
\langle G\rangle_{T}^{\prime}=\frac{24 \kappa G E \bar{\rho}^{3}}{24 E \bar{\rho}^{2}+\kappa G \pi^{2}}
$$

which is an expression analogous to that in (17). The quantities with the prime symbol ' denote properties of the staggered arrangement. For short overhang length $\lambda$, fibre shear could be significant. The modulus using EB assumptions ignoring fibre shear is $\langle G\rangle_{E B}^{\prime}=\left(24 / \pi^{2}\right) E \bar{\rho}^{3}$ and, therefore, an expression relating apparent shear moduli with and without fibre shear is obtained as

$$
\langle G\rangle_{T}^{\prime} \approx\langle G\rangle_{E B}^{\prime}\left(1-7.36 \bar{\rho}^{2}\right)+\mathrm{O}\left(\bar{\rho}^{4}\right)
$$

This expression is analogous to the one in equation (19) but for a different stacking arrangement. Because of the change of the effective length at which the filaments are in flexure, the apparent shear modulus of the staggered lattice material having the same relative density as the aligned arrangement, results in 4 
times stiffer leading correction factors $1.84 \bar{\rho}^{2}$ vs $7.36 \bar{\rho}^{2}$ as observed in equations (19) and 21 respectively. Note that the two models developed here under the assumption that the rolling between them is excluded because rotation of cylinders with circular cross-section in the $x-z$ plane are not allowed to rotate in that plane. In the next section, woodpile lattice materials modelled using FEA are validated against the analysis presented in Sections 2.4 and 2.5 .

\subsection{Validation of fibre shear corrected lattice shear modulus}

Analytical expressions for the apparent shear modulus obtained in Sections 2.4 and 2.5 are compared with numerical simulations using the finite element analysis (FEA) now. The apparent shear modulus obtained from unit cells of different relative densities are compared on a logarithmic scale in figure 9 The finite element model allowed lattice shear given purely by the transverse deflection of the struts, while constraining rolling of adjacent filaments. The scaling relation $\langle G\rangle \sim(\bar{\rho})^{3}$ is confirmed on a log-log plot by a slope very close to 3 . This is consistent with the bending dominance in the filaments assumed here. An excellent agreement between FEA numerical experiments and the analytical models developed in the previous subsections can be observed for lattices with low relative densities $(\bar{\rho}<0.3)$. For relatively denser lattices, Timoshenko's correction in the micromechanics of the filaments improves the agreement significantly whereas the Euler-Bernoulli (EB) model is inadequate. Despite significant improvements by the introduction of filament shear for dense scaffolds, some differences between analytical prediction and the FEA simulations remain. They are attributed to two main factors. First, for denser lattices $(\bar{\rho}>0.3)$, the filaments can no longer be modelled as thin beams, as they are now in a complex state of stress. The inset in figure 9 shows a comparison between the errors obtained from the analytical models using Euler-Bernoulli theories of the staggered and aligned configurations. Errors for filament separation equal to $\lambda / 2$ for the aligned arrangement are approximately the same when the separation is $\lambda$ for the staggered arrangement. This is expected from the lattice shear patterns shown in figures 7 and 8 , also confirmed numerically by the straight 
line at $45^{\circ}$ passing through the origin (see inset in figure 9). Secondly, there is always finite overlap between two orthogonally adhering cylinders, rather than at a single point, to ensure bonding of filaments in the finite element mesh. A simple flexural theory does not account for this. How the overlap influences the shear response will be further discussed in Section 3.2 .



Figure 9: Apparent shear modulus $\langle G\rangle$ for both woodpile arrangements as function of the relative density $\bar{\rho}$ and a comparison with computational experiments using FEA. The dotted lines correspond to the prediction using EB assumptions, while the solid lines correspond to the filament shear inclusive model.

\section{Numerical simulation and reconciliation with theoretical models}

Numerical simulations give us the opportunity to test models quickly for a large number of cases in a controlled and systematic manner, in order to be able to attribute certain physical effects to appropriate factors. With this advantage of computer simulations, finite element models of cantilever scaffold structures were subjected to transverse loading for a very large number of structures having a range of porosity, external length, number of stacks, stacking arrangements and filament overlap. These trends are then compared reconciled with theoretical developments presented in the previous section. The cantilever external geometry provides an ideal setting to test theoretical models, besides being typ- 
ical and also simple to implement while possessing all the essential features of the problem.

Commercial finite element code Abaqus/CAE/Standard 6.13 (Simulia, Dassault Systmes, Providence, RI, USA) [45] was used to numerically calculate the response of a lattice beam under transverse loading. The struts of the lattice were modelled as cylinders of diameter $d=0.5 \mathrm{~mm}$ and meshed using linear tetrahedral elements C3D10 (figure 10p). PLA material is commonly used in fused deposition modelling - the values of the properties chosen here are $E=2.29 \mathrm{GPa}, \nu=0.36$. Bonding between layers was represented by an overlapping region between the volumes of contacting cylinders that belong to adjacent layers. The overlap was systematically changed to study its influence on the resulting apparent properties - the results are reported in Section 3.2 . Other structural parameters, i.e. the overall length of the beam $L$, distance between the struts $\lambda$, and number of layers $N_{z}$, were also systematically changed to study their influence on the transverse deflection of cantilever beams.

When cylindrical filaments are placed orthogonally with a single point of adhesion, the joint is free to rotate in a numerical model. This is unrealistic for real material as those fabricated using 3D printing such as FDM (see figure 10p). Therefore, we reduced the distance between the axes of the two non-intersecting cylinder along the direction of closest approach, such that cylindrical surfaces dig into each other, resulting in a patch of overlap (the SEM micrograph shown in figure $10 \mathrm{~b}$ justifies the need for this). An overlap factor $O L$, is defined as the fraction of the cylinder diameter and the centre-to-centre distance $\lambda_{z}$ given by $O L=\left(2 r-\lambda_{z}\right) / 2 r$ (see figure $10 k$ ). $O L=8 \%$ is used in our simulations presented in Section 3.1 .

\subsection{Transverse response of woodpile beams: mechanism of macroscopic and} filament deformations

Solid beams in flexure show a deflection-length dependence as $\delta \sim L^{3}$ whereas a shear beam shows $\delta \sim L$. This observation could be used effectively to determine the relative importance of the two modes of deformation-apparent 




(a)

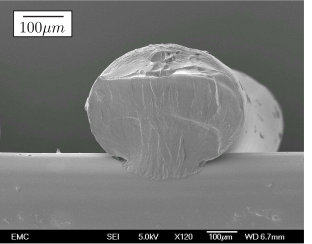

(b)

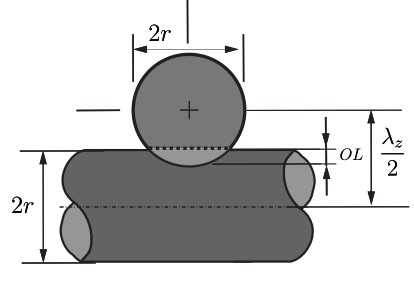

(c)

Figure 10: (a) A representative portion of the FEA models showing the loading direction and the mesh. (b) SEM micrograph of a FDM filament cross-section showing the bonding between layers. (c) Schematic diagram of the overlap of two adjacent filaments.

bending of the lattice which is associated with filament stretch and the apparent shear of the lattice which is associated with the filament bending within woodpile lattice materials. Further, any similarity or differences in the mechanical behaviour of a lattice beam with respect to those of a homogeneous continuum beam can be readily identified in this way.

A homogeneous cantilever beam of length $L$ under tip loading $F$ has tip deflection given by $\delta=F L^{3} /(3 B)$. For a beam made of porous material, $\delta=F L^{3} /(3\langle B\rangle)$, which shows $\delta \sim L^{3}$ scaling. Keeping all other lattice parameters the same, finite element models of porous beams of different length were subjected to tip loading and the tip deflection was calculated. The structure in each case was discretised using tetrahedral elements as described earlier. The tip deflection $(\delta / L)$ is plotted against the square of length of the lattice beam $L^{2}$ in figure 11 a for three different values of $N_{z}$, the number of layers in the stacking direction $z$. Each dot in this figure represents result for one structure. The straight line dependence validates $\delta \sim L^{3}$ relationship, with linear graphs of differing slopes-the difference being attributed to the differences in bending stiffness resulting from differing $N_{z}$.

As we have an analytical model for the apparent bending stiffness $\langle B\rangle$ in Section 2.3. which makes use of the micromechanics of filaments when the lattice beam is bent, we use the theoretical value of $\langle B\rangle$ from equations 10$\rangle, 11$ and 


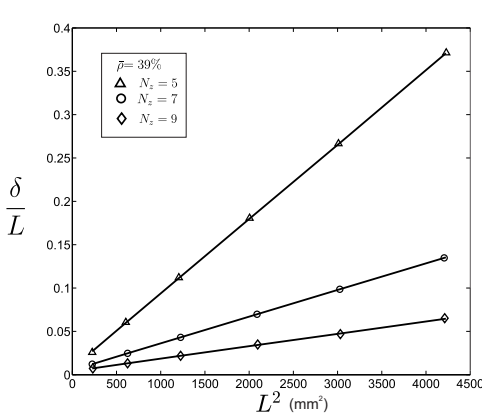

(a)

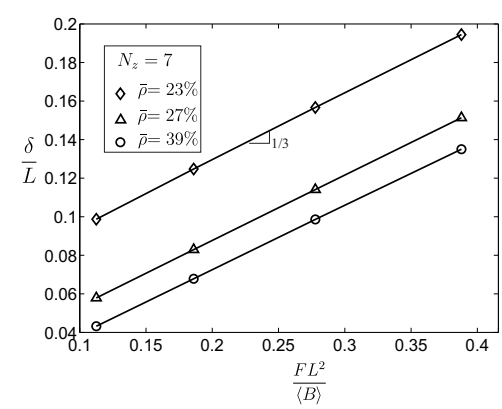

(c)



(b)

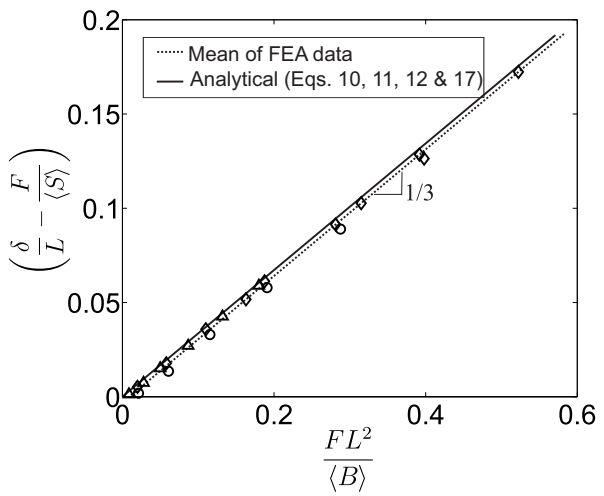

(d)

Figure 11: (a) Plot $(\delta / L)$ vs $L^{2}$, the lines presented here correspond to woodpile beams with different number of layers, $N_{z}=5, N_{z}=7$ and $N_{z}=9$. These lines have different slopes which means that different values $\langle B\rangle$ is different for all cases. (b) and (c) Plot $(\delta / L)$ vs $F L^{2} /\langle B\rangle$, the lines presented here have the same slope and the difference of their intercepts is due to the shear influence in each case. (d) Plot $(\delta / L-F /\langle S\rangle)$ vs $\left(F L^{2} /\langle B\rangle\right)$ showing the convergence of the data to a single line of slope $1 / 3$. The data here was calculated using equations 10, 111 and 12 for $\langle S\rangle$, and equation 17p for $\langle S\rangle$.

12 in the non-dimensional equation

$$
\frac{\delta}{L}=\frac{F L^{2}}{3\langle B\rangle}
$$

Therefore, all the data presented in figure $11 \mathrm{a}$ must collapse to a line on the $\frac{\delta}{L}$ vs $\frac{F L^{2}}{3\langle B\rangle}$ plane. This is indeed the case as seen from figure $11 \mathrm{~b}$ which also confirms the validity of expressions for $\langle B\rangle$ derived in Section 2.3 . Data in figure $11 \mathrm{~b}$ refer to response of 18 different porous structures when the number of layers 
and the overall length of the beam are changed systematically, while keeping the volume fraction $\bar{\rho}$ fixed at $39 \%$.

Next, the number of layers is fixed to $N_{z}=7$ and the porosity is controlled by changing the filament spacing as reflected by reducing $\bar{\rho}$ from $39 \%$ to $23 \%$. With increased porosity, data on the $\delta / L$ vs. $F L^{2} /(3\langle B\rangle)$ plane now separate into three distinct groups. The (1/3)-rd slope confirms quantitatively accurately predictable beam bending behaviour, however the shift in the intercept for varying porosity indicates missing aspects of mechanics from equation 22. . Moreover, this plot also suggests that the missing mechanics must provide $\delta / L=$ constant dependence, i.e. $\delta \sim L$ for the deflection unaccounted by equation (22). This scaling with the beam overall length $L$ is consistent with shear deformation. Thus the relationship that could collapse all data for any lattice geometry involving a range of filament diameters, material, filament spacing, number of layers, or overall length of the beam must be given by a beam deflection formula that accounts for the apparent bending as well as the apparent shear of the lattice beam. Note that the apparent bending of lattice beam is associated primarily with filament stretch along the length of the beam, whereas the apparent shear of the lattice beam is associated primarily with the filament bending. In solid beams, the relative importance of stretch and shear deformations are interdependent because they scale with the inverse of the Young's modulus and the shear modulus of the material respectively. By contrast, for lattice beams, the apparent shear modulus depends on the Young's modulus of the filament material and the architecture of the lattice in its leading order behaviour. The shear modulus of the filament material has only a secondary role via the shear correction of the the filament deflection.

The formula for the tip deflection of a cantilevered beam loaded at the end accounting for shear is $\delta=F\left(L^{3} / 3 B+L / S\right)$. This expression can also be presented in the following non-dimensional form for a lattice beam with 
apparent properties $\langle B\rangle$ and $\langle S\rangle$ as

$$
\frac{\delta}{L}=F\left(\frac{L^{2}}{3\langle B\rangle}+\frac{1}{\langle S\rangle}\right)
$$

Comparing the above equation with equation 22 , we note that an additional apparent property $\langle S\rangle=\kappa\langle G\rangle A$ is now required.

The dominant scaling behaviour for the apparent shear modulus is given by $\langle G\rangle \sim(r / \lambda)^{3} \sim(\bar{\rho})^{3}$ because $(\bar{\rho}) \sim(r / \lambda)$ after retaining the leading order terms in equations 17 and 20 for the two woodpile configurations respectively. Thus porosity $\bar{\rho}$ has a dramatic effect on the transverse response. The transverse response as shown in figure 11 differs by a huge factor of about 12, i.e. $1200 \%$ for short beams (left end of the plot) when porosity porosity reduces from $39 \%$ to $23 \%$ highlighting the significance of consideration to shear for porous woodpile lattices.

The apparent shear stiffness is associated with the flexure of the filament, as noted in Section 2.4 and is given by $\langle S\rangle=\kappa\langle G\rangle A$, where $\kappa=5 / 6$ for rectangular cross-sections 44 and where $\langle G\rangle$ is given by equation (17). When we include shear flexibility due to filament flexure in the model, data from low porosity (as in figure $11 \mathrm{~b}$, where shear is insignificant and an apparent-flexure-only model adequate) to relatively high porosity data (as in figure 11c), collapse to a single line (figure 11d) when the deflection associated with shear $\delta_{\text {shear }} / L=F /\langle S\rangle$ is subtracted. Different marker types in figure $11 \mathrm{~d}$ correspond to tip response for different geometries when the aspect ratio of the overall cantilever beam and the porosity are changed. Note that the shear stiffness $\langle S\rangle$ used to plot these markers are obtained using the apparent shear modulus $\langle G\rangle$ from analytical expressions (17).

We now compare the transverse elastic response of woodpile beams in the plane of deflection for the two configurations considered here in figure $12 \mathrm{a}$. The top beam is composed of the aligned arrangement while the bottom one is a staggered structure. The deformed shapes of figure $12 \mathrm{a}$ were obtained from FEA simulations. These two examples are expected to have significant differences due 


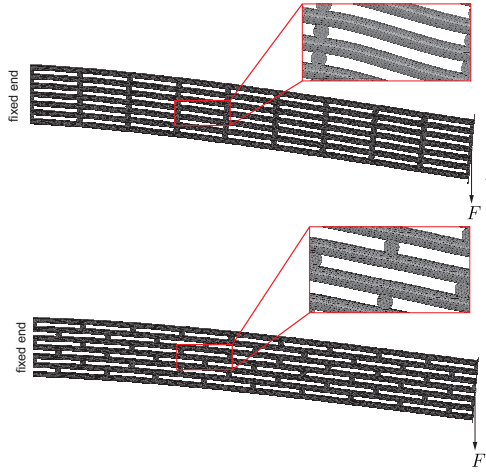

(a)

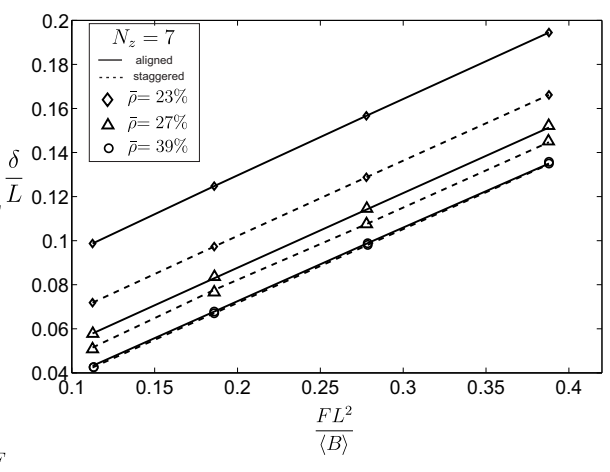

(b)

Figure 12: (a) Deformed shapes from FEA simulations of cantilever beams composed of the two woodpile arrangements: woodpile aligned (top) and woodpile staggered (bottom). Both beams have are composed of lattice materials with the same relative density and have the same number of axial struts, resulting on the the same bending stiffness. The staggered architecture presents higher resistance to shear, as expected. (b) Comparison between the data obtained from FEA

to the role of shear in the transverse deflection in bending, as both beams have the same effective bending stiffness and relative density (the spacing between the struts $\lambda$ is the same). The data for several beams with 7 layers, and various relative density $(\bar{\rho}=23 \%, \bar{\rho}=27 \%$ and $\bar{\rho}=39 \%)$ is plotted on the $\frac{\delta}{L}$ vs $\frac{F L^{2}}{3\langle B\rangle}$ plane in where all lines appear parallel in figure $12 \mathrm{~b}$. The role of shear is reflected in the different intercepts obtained, which are more significant for beams with high porosity. For dense beams this effect is negligible. Note in figure 12 that for beams with the same relative density, the aligned configuration shows greater intercepts, which means that the apparent shear modulus is lower than that for the staggered configuration. The shear stiffness is found to be sensitive to the overlap of filaments at the region of adhesion. This is discussed next.

\subsection{Sensitivity to overlap}

Schematic diagrams in figures $7 \mathrm{a}$ and $8 \mathrm{a}$ show filaments bonded at a single point. In real manufacturing scenarios [34, 42], this is not observed. We printed samples of lattice material using FDM (nozzle diameter of $0.5 \mathrm{~mm}$, and PLA material) and cross-section of the samples were then observed using SEM (figure 10b). The image presented in figure $10 \mathrm{~b}$ confirms significant overlap. 
A single point joint between two contacting cylindrical filaments offers no shear resistance in a computational model even when the nodes belonging to different cylinders are tied up via translational degrees of freedom. This behaviour is very similar to a pile of elastic sheets (such as a stack of sheets of paper), which when bent slides over each other without much shear resistance. For woodpile materials, adjacent layers "roll" over each other at "hinges" provided by tie points and the only elastic resistance is provided by deforming contacting surfaces which requires careful implementation of this aspect of contact within commercial FE codes.

The sensitivity of the overlap factor $O L$ is systematically studied computationally and is presented in figure 13 As the overlap increases, the apparent shear stiffness $\langle S\rangle$ increases and saturates to the theoretical value of $\kappa\langle G\rangle A$ where $\langle G\rangle$ is given by the formula developed in Section 2.4 . The value of $\langle S\rangle$ is interpreted from the intercepts of lines in figure $11 \mathrm{c}$. The trend can be explained as the theoretical values are based on shear deformation that constraints rotation of the filaments rolling over each other. Thus theoretical values of $\kappa\langle G\rangle A$ provide upper limit to the shear stiffness, when the overlap factor is increased.

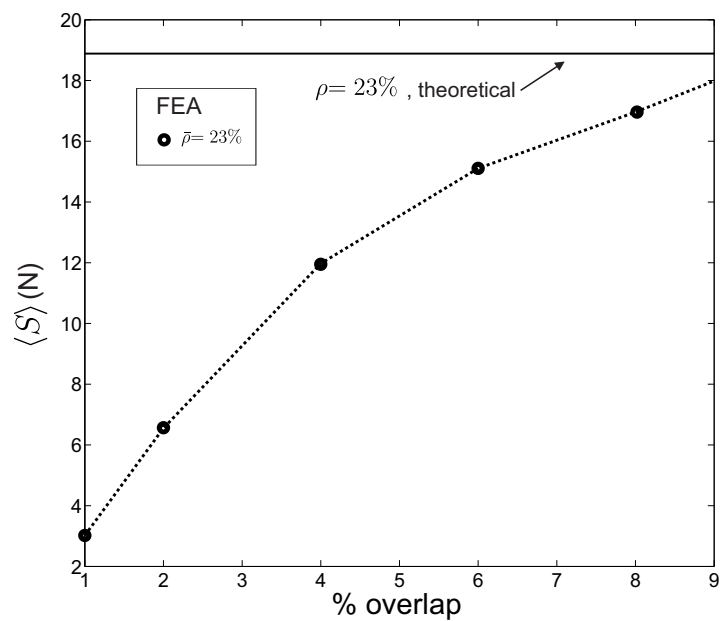

Figure 13: Convergence of the value for the $\langle S\rangle$ as the $\%$ overlap is incremented. The incrementation of the overlap will make the shear response to be completly dominated by the bending of the struts. This value is presented with dotted line. 


\section{Conclusions}

Analytical expressions for the apparent elasticity of structured materials with woodpile architecture were developed when slender lattice structures experience apparent stretch, bending and shear. When a slender beam made up of material with such microstructure is bent, the filaments running parallel to the axis of the beam experience stretch primarily, whereas when the beam is sheared, the filaments experience bending. This fundamental micromechanics of the filaments enables us to derive analytical expressions for finite number of stacks of the woodpile and also the dependence of bending and shear stiffness in terms of the relative density of the porous structure. Experimental results from three-point-bending tests on additively manufactured structures are in excellent agreement with analytical predictions. Analytical results, presented for the first time, are also expressed in terms of asymptotic formula for large number of layers. The expressions thus derived indicate that the bending stiffness scales with the relative density as $\sim \bar{\rho}$ whereas the shear stiffness scales as $\sim \bar{\rho}^{3}$ which is consistent with the primary mechanisms of filament deformation identified for these two respective cases of transverse deflection of the structure. The first of these is also consistent with the apparent Young's modulus scaling as $\langle E\rangle \sim \rho$. Derivations from $\langle G\rangle \sim \rho^{3}$ for denser lattices are successfully dealt by including shear within the filaments as further refinement to the model.

For relatively dense lattices and for long scaffolds bending, a filament stretch based apparent-bending-only-model, which ignores lattice shear, provides satisfactory agreement with numerical results. However, when the lattice is relatively porous and the overall structure relatively short, ignoring lattice shear leads to

huge errors-up to $1200 \%$ for the worst cases considered. Such large influence of shear is a reflection of the lattice microstructure: dense elastic continuum beams require much smaller correction than porous beams rending the problem of lattice beams one where shear is not a mere correction but it needs to be an essential part of the model, the relative significance increasing with porosity. When lattice spacing is large, significant lattice shear needs to be included 
in the model in addition to bending. When the lattice spacing is small, filament shear needs to be included in addition to combined filament stretch and bending. An excellent quantitative agreement between shear-inclusive bending theory developed here and numerical computation for a large range or porosity and slenderness of the beam is reached-this is demonstrated by identifying a shear adjusted non-dimensional group which collapses all elastic response data for lattice beam aligned to a theoretically predicted trend.

Expressions for apparent bending stiffness $\langle B\rangle$ and the apparent shear stiffness $\langle S\rangle$ are derived for two configurations of the woodpile-first when the alternating layers eclipes each other and the latter when they are staggered. The bending stiffness is independent of the configuration whereas the shear stiffness shows strong differences: for the same porosity, the staggered arrangement is approximately 4 times as stiff in shear. Finally, the role of the so-called overlap factor is brought out which indicates that the stiffness has a upper bound given by the analytical expressions which constrain rotation of cylinder over each other. In summary, we provide benchmark results for transverse response of slender lattice beams with excellent agreement with a shear-inclusive model which uses apparent bending and shear stiffness, also provided analytically here.

Acknowledgements Financial support from CONACyT (Mexican Council) for a $\mathrm{PhD}$ degree and also British Council UKIERI grant are gratefully acknowledged.

\section{References}

[1] C. R. Tubio, J. Azuaje, L. Escalante, A. Coelho, F. Guitián, E. Sotelo, A. Gil, 3d printing of a heterogeneous copper-based catalyst, Journal of Catalysis 334 (2016) 110-115.

[2] C. Hurt, M. Brandt, S. Priya, T. Bhatelia, J. Patel, P. Selvakannan, S. Bhargava, Combining additive manufacturing and catalysis: a review, Catalysis Science \& Technology 7 (16) (2017) 3421-3439. 
[3] J.-H. Lee, Y.-S. Kim, K. Constant, K.-M. Ho, Woodpile metallic photonic crystals fabricated by using soft lithography for tailored thermal emission, Advanced Materials 19 (6) (2007) 791-794.

[4] A. R. Weily, K. P. Esselle, B. C. Sanders, T. S. Bird, A woodpile ebg sectoral horn antenna, in: Antennas and Propagation Society International Symposium, 2005 IEEE, Vol. 4, IEEE, 2005, pp. 323-326.

[5] X. Lu, Y. Lee, S. Yang, Y. Hao, J. Evans, C. Parini, Extrusion freeforming of millimeter wave electromagnetic bandgap (EBG) structures, Rapid Prototyping Journal 15 (1) (2009) 42-51.

[6] J. Stuecker, J. Cesarano, J. Miller, Regenerable particulate filter, US Patent $7,527,671(2009)$. URL https://www.google.com/patents/US7527671

[7] D. W. Hutmacher, Scaffolds in tissue engineering bone and cartilage, Biomaterials 21 (24) (2000) 2529-2543.

[8] S. Michna, W. Wu, J. A. Lewis, Concentrated hydroxyapatite inks for direct-write assembly of 3-D periodic scaffolds, Biomaterials 26 (28) (2005) $5632-5639$.

[9] J. A. Lewis, Direct ink writing of 3D functional materials, Advanced Functional Materials 16 (17) (2006) 2193-2204.

[10] J. M. Sobral, S. G. Caridade, R. A. Sousa, J. F. Mano, R. L. Reis, Threedimensional plotted scaffolds with controlled pore size gradients: effect of scaffold geometry on mechanical performance and cell seeding efficiency, Acta Biomaterialia 7 (3) (2011) 1009-1018.

[11] S.-H. Ahn, M. Montero, D. Odell, S. Roundy, P. K. Wright, Anisotropic material properties of fused deposition modeling ABS, Rapid prototyping journal 8 (4) (2002) 248-257. 
[12] M. Montero, S. Roundy, D. Odell, S.-H. Ahn, P. K. Wright, Material characterization of fused deposition modeling (FDM) ABS by designed experiments, Society of Manufacturing Engineers 10 (13552540210441166).

[13] B. Tymrak, M. Kreiger, J. M. Pearce, Mechanical properties of components fabricated with open-source 3-D printers under realistic environmental conditions, Materials \& Design 58 (2014) 242-246.

[14] S. Brischetto, C. G. Ferro, P. Maggiore, R. Torre, Compression tests of abs specimens for uav components produced via the fdm technique, Technologies 5 (2) (2017) 20.

[15] A. Bellini, S. Güçeri, Mechanical characterization of parts fabricated using fused deposition modeling, Rapid Prototyping Journal 9 (4) (2003) 252264.

[16] M. Too, K. Leong, C. Chua, Z. Du, S. Yang, C. Cheah, S. Ho, Investigation of 3D non-random porous structures by fused deposition modelling, The International Journal of Advanced Manufacturing Technology 19 (3) (2002) $217-223$.

[17] A. K. Sood, R. K. Ohdar, S. S. Mahapatra, Parametric appraisal of mechanical property of fused deposition modelling processed parts, Materials \& Design 31 (1) (2010) 287-295.

[18] G. C. Onwubolu, F. Rayegani, Characterization and optimization of mechanical properties of abs parts manufactured by the fused deposition modelling process, International Journal of Manufacturing Engineering 2014.

[19] W. Wu, P. Geng, G. Li, D. Zhao, H. Zhang, J. Zhao, Influence of layer thickness and raster angle on the mechanical properties of 3D-printed PEEK and a comparative mechanical study between PEEK and ABS, Materials 8 (9) (2015) 5834-5846.

[20] D. Croccolo, M. De Agostinis, G. Olmi, Experimental characterization and analytical modelling of the mechanical behaviour of fused deposition pro- 
cessed parts made of ABS-M30, Computational Materials Science 79 (2013) 506-518.

[21] M. Yeo, C. G. Simon, G. Kim, Effects of offset values of solid freeform fabricated pcl- $\beta$-TCP scaffolds on mechanical properties and cellular activities in bone tissue regeneration, Journal of Materials Chemistry 22 (40) (2012) 21636-21646.

[22] J. Norato, A. W. Johnson, A computational and cellular solids approach to the stiffness-based design of bone scaffolds, Journal of biomechanical engineering 133 (9) (2011) 091003.

[23] S. Naghieh, M. K. Ravari, M. Badrossamay, E. Foroozmehr, M. Kadkhodaei, Numerical investigation of the mechanical properties of the additive manufactured bone scaffolds fabricated by FDM: The effect of layer penetration and post-heating, Journal of the mechanical behavior of biomedical materials 59 (2016) 241-250.

[24] T. Serra, J. A. Planell, M. Navarro, High-resolution pla-based composite scaffolds via 3-D printing technology, Acta biomaterialia 9 (3) (2013) 55215530.

[25] J. Cesarano, J. G. Dellinger, M. P. Saavedra, D. D. Gill, R. D. Jamison, B. A. Grosser, J. M. Sinn-Hanlon, M. S. Goldwasser, Customization of loadbearing hydroxyapatite lattice scaffolds, International Journal of Applied Ceramic Technology 2 (3) (2005) 212-220.

[26] F. J. Martínez-Vázquez, P. Miranda, F. Guiberteau, A. Pajares, Reinforcing bioceramic scaffolds with in situ synthesized $\varepsilon$-polycaprolactone coatings, Journal of Biomedical Materials Research Part A 101 (12) (2013) $3551-3559$.

[27] F. J. Martínez-Vázquez, A. Pajares, F. Guiberteau, P. Miranda, Effect of polymer infiltration on the flexural behavior of $\beta$-tricalcium phosphate robocast scaffolds, Materials 7 (5) (2014) 4001-4018. 
[28] X. Liu, M. N. Rahaman, G. E. Hilmas, B. S. Bal, Mechanical properties of bioactive glass (13-93) scaffolds fabricated by robotic deposition for structural bone repair, Acta biomaterialia 9 (6) (2013) 7025-7034.

[29] S. Eqtesadi, A. Motealleh, A. Pajares, F. Guiberteau, P. Miranda, Improving mechanical properties of 13-93 bioactive glass robocast scaffold by poly (lactic acid) and poly ( $\varepsilon$-caprolactone) melt infiltration, Journal of Non-Crystalline Solids 432 (2016) 111-119.

[30] M. Domingo-Espin, J. M. Puigoriol-Forcada, A.-A. Garcia-Granada, J. Lluma, S. Borros, G. Reyes, Mechanical property characterization and simulation of fused deposition modeling polycarbonate parts, Materials \& Design 83 (2015) 670-677.

[31] M. Malinauskas, E. Skliutas, L. Jonušauskas, D. Mizeras, A. Šešok, A. Piskarskas, Tailoring bulk mechanical properties of 3D printed objects of polylactic acid varying internal micro-architecture, in: SPIE Optics+ Optoelectronics, International Society for Optics and Photonics, 2015, pp. 95050P-95050P.

[32] L. J. Gibson, M. F. Ashby, Cellular solids: structure and properties, Cambridge university press, 1999.

[33] P. Moongkhamklang, D. M. Elzey, H. N. Wadley, Titanium matrix composite lattice structures, Composites Part A: applied science and manufacturing 39 (2) (2008) 176-187.

[34] P. Moongkhamklang, V. Deshpande, H. Wadley, The compressive and shear response of titanium matrix composite lattice structures, Acta Materialia 58 (8) (2010) 2822-2835.

[35] S. Banerjee, A. Bhaskar, Free vibration of cellular structures using continuum modes, Journal of sound and vibration 287 (1) (2005) 77-100. 
[36] S. Banerjee, A. Bhaskar, The use and limitations of continuum modes for response calculations of cellular structures, Journal of sound and vibration 302 (3) (2007) 457-470.

[37] S. Banerjee, A. Bhaskar, The applicability of the effective medium theory to the dynamics of cellular beams, International Journal of Mechanical Sciences 51 (8) (2009) 598-608.

[38] E. Cuan-Urquizo, A. Bhaskar, Apparent Young's modulus of the woodpile lattice material along the stacking direction, manuscript in preparation.

[39] S. P. Timoshenko, Lxvi. on the correction for shear of the differential equation for transverse vibrations of prismatic bars, The London, Edinburgh, and Dublin Philosophical Magazine and Journal of Science 41 (245) (1921) 744-746.

[40] A. Bhaskar, Elastic waves in timoshenko beams: the lost and foundof an eigenmode, in: Proceedings of the Royal Society of London A: Mathematical, Physical and Engineering Sciences, Vol. 465, The Royal Society, 2009, pp. 239-255.

[41] P. Miranda, A. Pajares, E. Saiz, A. P. Tomsia, F. Guiberteau, Fracture modes under uniaxial compression in hydroxyapatite scaffolds fabricated by robocasting, Journal of Biomedical Materials Research Part A 83 (3) (2007) 646-655.

[42] E. Cuan-Urquizo, S. Yang, A. Bhaskar, Mechanical characterisation of additively manufactured material having lattice microstructure, in: IOP Conference Series: Materials Science and Engineering, Vol. 74, IOP Publishing, 2015, p. 012004.

[43] M. Jamshidian, E. A. Tehrany, M. Imran, M. Jacquot, S. Desobry, Polylactic acid: production, applications, nanocomposites, and release studies, Comprehensive Reviews in Food Science and Food Safety 9 (5) (2010) 552571. 
[44] J. M. Gere, S. Timoshenko, Mechanics of materials brooks, Cole, Pacific Grove, CA (2001) 815-39.

[45] D. SYSTEMES, Abaqus 6.11 analysis users manual, Online Documentation Help. 